\title{
A qualitative study exploring patient, family carer and healthcare professional perceptions of providing palliative care for advanced head and neck cancer
}

Catriona Rachel Mayland ( $\sim$ C.R.Mayland@sheffield.ac.uk)

University of Liverpool https://orcid.org/0000-0002-1440-9953

Hannah Doughty

Clatterbridge Cancer Centre NHS Foundation Trust

Simon Rogers

Edge Hill University

Anna Gola

UCL

\section{Stephen Mason}

University of Liverpool

Cathy Hubbert

Aintree Park GP Practice

Dominic Macareavy

Aintree University Hospitals NHS Foundation Trust

Barbara Jack

Edge Hill University

Research article

Keywords: Head and neck cancer, Palliative care, Integration, Qualitative research, Interviews

Posted Date: July 3rd, 2019

DOI: https://doi.org/10.21203/rs.2.10855/v1

License: @) (7) This work is licensed under a Creative Commons Attribution 4.0 International License. Read Full License 


\section{Abstract}

Purpose: To explore patient, family carer and healthcare professionals' perspectives about providing palliative care for advanced head and neck cancer patients and potential components which could inform a future model of care. Methods: Using a naturalistic, interpretative approach, within Northwest England, a purposive sample of adult head and neck cancer patients was selected. Their family carers were also invited to participate. Healthcare professionals (representing head and neck surgery and specialist nursing; oncology; specialist palliative care; general practice and community nursing) were recruited. All participants underwent face-to-face or telephone interviews. A thematic approach, using a modified version of Colazzi's framework, was used to analyse the data. Results: In total, 17 interviews were conducted (nine patients, four joint with family carers and eight healthcare professionals). Three main themes relating to challenges in providing palliative care were identified: the 'need for support at critical moments' in the patients' disease trajectory; 'communication challenges' and 'complexity of decision-making'. Additionally, three themes related to key components to inform a future model of care: 'partnership model'; 'timely and responsive engagement of Specialist Palliative Care'; and 'good linkage and connections'. Conclusions: Conceptually, the identified 'critical moments' in the patients' disease trajectory should be a focus for future interventions to help improve and support care. These interventions should direct improvements in the provision of information and psychological support. Models of palliative care for this population need to be developed in partnership with existing services to ensure care is delivered in a coordinated and timely manner.

\section{Introduction}

Globally, head and neck cancer is the sixth most common cancer with over 550,000 people diagnosed annually [1]. Head and neck cancer (HNC) and its treatment affects patients' ability to communicate, swallow and breathe as well as the resultant physical disfigurement and psychological issues [2-5]. Patients have a higher incidence of depression and a disproportionately higher risk of suicide compared with the general cancer population [6]. Family members also report distress and unmet needs when supporting those with HNC [7-9].

The beneficial impact of integrating palliative care early into routine oncological care is well established in numerous good quality clinical studies [10-15]. Correspondingly, the American Society of Clinical Oncology recommends advanced cancer patients within the in-patient and out-patient setting should receive palliative care services early in their disease trajectory, often alongside active treatment [16]. The European Society for Medical Oncology [17] and World Health Organisation advocate similar recommendations. The optimal way to 'incorporate palliative care in the multidisciplinary management of patients with high risk squamous cell cancer of the head and neck however, remains unclear [18]. Questions remain about the ideal mode of delivery, the optimal timing for referral and ways to identify patients with the greatest needs to ensure effective, efficient and sustainable services [10]. This is especially pertinent when considering the different systems and structures of healthcare provision.

Within the United Kingdom (UK), HNC care is based on a centralised multidisciplinary model with service integration advocated via a 'key worker' role and usually facilitated by a specialist HNC nurse [19]. National recommendations advise all professionals caring for HNC patients assess palliative and supportive care needs throughout the illness, including at initial treatment planning, and recognise when Specialist Palliative Care (SPC) expertise is required [20]. Often HNC Multi-Disciplinary Team (MDT) meetings are not able to provide direct SPC attendance which may impact on quality of patient care [19].

Within the context of an illness with high complexity, the aim of this study was to explore, from the perspective of the patient, family carer, and healthcare professional, the current challenges in providing palliative care for people with advanced HNC. Additionally, we wanted to investigate potential service model components which could enhance palliative care integration. In order to achieve these aims, a methodological approach was selected drawing upon a naturalistic, interpretative approach [21]. This enabled an in-depth and rich understanding of the participants' experiences and perceptions [22].

\section{Methods}

\section{Study setting}

One of the largest national HNC MDT meetings occurs weekly within Northwest England. All new and recurrent cancer patients are discussed, equating to an average of 70 patients per month (approximately eight per month treated with palliative intent). Regional SPC services, funded through public and charitable sources, are provided by separate MDTs who have undergone specific training to develop expert skills. Although SPC teams have different structures, national recommendations advise they should contain a senior physician, a specialist nurse and, at minimum, have direct input from a wide range of allied healthcare practitioners (such as physiotherapy, occupational therapy, spiritual and psychological services and social workers) to help provide holistic, person-centred care [23]. Regionally, they are able to provide advisory input within the community setting (at home, care home or via out-patients) working in collaboration with other care givers such as the General Practitioners and District Nurses (community doctors and nurses respectively). Advisory input can be provided within acute hospitals or the SPC team can be directly responsible for care for patients within a SPC in-patient unit or hospice.

\section{Participant selection}

Patients and family carers

Between May and November 2017, a purposive sample of adult patients (over 18 years old) was obtained according to the following criteria [24]:

Histological or radiological diagnosis of 'advanced' HNC

Fully aware of their diagnosis 
Able to provide informed consent.

The term 'advanced' HNC incorporated those treated with palliative intent, and those treated with curative intent but whom the clinical team judged were 'high risk' for developing recurrent disease. The sample was purposive to try to seek views from a variety of patients in terms of gender, age group and primary site of cancer. Additionally, we wanted to include those currently engaged with SPC services or those who would potentially most benefit from early palliative care intervention. Those unable to provide informed consent, perceived to be unduly distressed by participation (by the clinical or research team) or who lived out with the northwest region (and hence would be burdensome for them to attend a face-to-face interview) were excluded.

Potential patients, meeting the inclusion criteria, were identified by members of the clinical teams during the conduct of the weekly HNC MDT meetings. Additional identification of potential participants was via out-patient clinics and SPC services in hospitals and hospices. Initial project information and a Participant Information Sheet was provided by the clinical team. Where the potential participant was willing to speak with the research team, contact details were passed on via the clinical team, and a research team member made contact either face-to-face or via telephone. The opportunity for further information and questions was provided. Where possible, for each potential patient, the family carer was identified and asked if they wished to participate.

Health care professionals

Potential participants were identified using a 'word-of-mouth' snowball sampling strategy as this is recognised to benefit 'inductive, theory-building analysis' [25]. Additionally, the potential challenges identifying community healthcare professionals were recognised. Hence, existing linkages with other members of the HNC MDT were sought to help with this identification. The initial sample was stratified to gain opinion from at least one clinical representative (minimum five interviews) working in the areas of HNC Surgery; Oncology; SPC; General Practice and Community Nursing. We reviewed this strategy during the study, and deemed that exploring HNC Clinical Nurse Specialist experiences would further enrich the data. This was in keeping with the concept of 'information power' [26], where items such as the study aim and sample specificity impact on the level of information a sample will hold. This approach offers an alternative to the 'saturation concept' traditionally associated with Grounded Theory [26]. Those who expressed a willingness to know more about the project, either made direct contact with the research team (through information provided on research posters) or passed on their details via existing participants. All received a Participant Information Sheet.

\section{Informed consent}

Informed consent was obtained from all individual participants included in the study.

Written consent was obtained to conduct an interview (and use anonymised quotations in publications), collect demographic data and additionally, for patients, to collect information about their cancer.

\section{Data collection}

Patient data were collected from case records and included demographic details (gender, age); Eastern Cooperative Oncology Group (ECOG) performance status [26]; primary diagnosis; whether metastatic disease was present; and treatment intent. Family carer data included gender and relationship to patient. Healthcare professional details included gender; age; length of time working as a healthcare professional and current area of practice.

\section{Interviews}

Semi-structured digitally recorded interviews were conducted in a place convenient for the patient and/or family carer (own home, hospital or hospice). For healthcare professionals, interviews were either conducted face-to-face or via digitally recorded telephone interviews (the latter offering a logistical, flexible solution to those who live across a wider geographical area) [27, 28].

All interviews were conducted by a researcher (HD) between June and November 2017. Methods to assist with communication were sought to aid participation. For two patients, where verbal communication was especially challenging, alternative means via written communication were used to supplement the interview (and directly checked with the participant for accuracy). Patient and family carer interviews ranged from 8 minutes to 114 minutes, mean 45 minutes; healthcare professional interviews ranged between 23 and 55 minutes, mean 39 minutes. Field notes were captured immediately after the interviews and a reflective diary was used to record immediate thoughts and impressions.

Exploratory topics within the interview schedule included (Supplementary file 1):

caring for a family member / patient with the illness.

Specific challenges and difficulties in care / providing palliative care.

Ways in which services could work differently to improve care.

Thoughts and feelings about earlier involvement of palliative care.

Initial interviews were reviewed and discussed by two members of the research team with questions refined as needed to explore issues in greater depth within future interviews.

Page $3 / 9$ 


\section{Analysis}

Demographic data were analysed descriptively to provide contextual information about the sample population. The recorded interviews were transcribed verbatim, anonymised during this process, thus removing identifying features. For the two patients where verbal communication was especially challenging, a written record was documented by the researcher. A thematic approach to analysis was conducted using the modified principles of Colazzi's framework [29], namely: organisation; familiarisation; reduction; and analysis. Although participant involvement at the analysis stage is of great value, this was not deemed to be practical, due to the advancing nature of the participants' illness and the limitation in terms of time available for the healthcare professionals.

To enhance rigour, two researchers (HD and CM) independently analysed each transcript to familiarise themselves with the data and recorded initial analytical notes or impressions. Field notes were checked where appropriate. An inductive approach to coding was used. Both researchers met to compare initial analysis and group the codes together into categories. The two main research questions, challenges to providing palliative care and potential service components to enhance care integration, were subsequently used as a framework for charting further analysis. Additional discussion, data reduction and analysis across cases was conducted in conjunction with a third researcher (BJ). A final coding scheme was agreed by the team, leading to identification of themes and subthemes. Checks against transcripts were used to assist with rigour.

\section{Results}

Participants

From 38 potentially eligible patient participants, ten agreed to be interviewed (Figure 1). One patient died prior to the interview being arranged, resulting in a sample of nine patients. It is noteworthy that an additional 11 patients died prior to contact or consent being obtained. Most of the participating patients were male $(n=7)$ and aged between 57 and 88 years. There was a wide range of different cancer sites (oropharynx, hypopharynx, tongue, mandible, and parotid gland). Four participants had distant metastatic disease (Table 1). Four family carers consented to a joint interview, three of whom were female, all were the patients' spouse. These joint interviews were all conducted in the patients' own home (Table 1). The remaining family members either could not be identified or declined participation.

Eight healthcare professionals were interviewed, with an equal gender split and representing the different chosen clinical areas (Table 2). Their length of time working in healthcare ranged from 15-32 years.

All participants were allocated a unique identifier code with participants' quotes used to support findings. For the two patients (P1 and P5), where verbal communication was challenging, written communication was incorporated to supplement the interview, and this is indicated by an asterix $(*)$.

Three main themes relating to challenges in providing palliative care were identified, namely: the need for support at 'critical moments' in the patients' disease trajectory; communication challenges and complexity of decision-making (Table 3). Additionally, there were three themes relating to potential service model components to enhance the integration of palliative care - partnership model; timely and responsive engagement of Specialist Palliative Care (SPC); and good linkage and connections (Table 4). A summary of these findings is presented below with the main themes, clarifying description and supporting participant quotes shown within the tables.

\section{[INSERT TABLE 3. THEMES RELATING TO CURRENT CHALLENGES IN PROVIDING PALLIATIVE CARE]}

Challenges in providing palliative care (Table 3)

\section{Support at 'critical moments' in the patients' trajectory}

Conceptually, patients, family carers and healthcare professionals perceived two main time periods when additional support was needed. These were prior to undergoing specific treatments and during the transition from curative treatment to incurable disease. Prior to specific treatments, both patients and family carers, reported additional information would have been helpful. Alternatively, they couldn't recall being given particular information. One patient wrote that he hadn't been aware he was going to have a tracheostomy (P5*). For another, their expectations and the subsequent reality about what the treatment would achieve were very different. Although the patient knew he was 'terminal' $\left(\mathrm{P} 1^{\star}\right)$, he thought he would feel 'better' after his operation and that the pain would have resolved. When being told their illness was incurable, the importance of family presence was highlighted as well asthe need for 'an additional appointment' $\left(\mathrm{P} 1^{*}\right)$ to allow for further discussion and support. During both time periods, healthcare professionals perceived that additional psychological support was needed.

\section{Communication challenges}

Healthcare professionals reported challenges in communication between different teams. They perceived this related to lack of natural opportunities for direct face-to-face working, especially between SPC services and the wider HNC MDTs. Additionally, misconceptions about hospice and palliative care, were reported by patients, family members and healthcare professionals and regarded as a prevalent problem.

\section{Complexity of decision making}

Decisions about the optimum treatment for an individual with HNC were often intricate and difficult as reflected by several different healthcare professionals. With the benefit of hindsight, reflections shared by patients, family members and healthcare professionals showed contemplation about whether or not the decision had been the 'right' one. 
Potential service model components to enhance palliative care integration (Table 4)

Partnership model

Healthcare professionals perceived integrated services were key for future developments, with a direct, more tangible presence from SPC. Additionally, 'patient information sheets for palliative care, or where they are going to be sent' (HCP1) were regarded as a method to more clearly signpost to specific clinical services. The provision of clinical treatment plans or 'management plans' (HCP2) were thought to potentially help integrate care better, especially in the context of improving the continuity of care across different settings e.g. from hospital to the community.

Timely and responsive engagement of Specialist Palliative Care (SPC)

It was recognised by patients, family carers and healthcare professionals that timely referral could facilitate good communication and help achieve patients' goals and wishes. Additionally, from one family carer (FC9), it was evident that appropriate engagement with SPC had helped provide a level of security which was flexible depending on needs.

\section{Good linkage and connections}

From the patients' perspective, having a key contact person was seen as ideal but somewhat aspirational. There was specific mention to the pivotal role of the Clinical Nurse Specialist by other healthcare professionals in terms of being a key contact and helping facilitate linkage between different teams.

[INSERT TABLE 4. THEMES RELATING TO POTENTIAL SERVICE MODEL COMPONENTS TO ENHANCE PALLIATIVE CARE INTEGRATION]

\section{Discussion}

Summary of main findings

Conceptually, patients, family carers and healthcare professionals perceived there were two 'critical moments' in the patient's disease trajectory when additional support was required. The first was during the decision-making period prior to having specific treatments, which was recognised as an especially complex and difficult time. Tailoring information to meet individual needs and providing sufficient psychological support at this time were recognised important factors to enhance informed decision-making and care. The second 'critical moment' was when the patient transitions from curable to incurable disease. The lack of integrated clinical services between SPC and the wider HNC MDT was perceived by healthcare professionals to hamper coordination of individualised care. Moving forward, potential service model components could include a partnership approach between the HNC and SPC teams. Potentially, this would help enhance integration to occur in a more suitable and coordinated manner.

How this study relates to existing literature

The importance of timely identification of patients who may benefit from palliative care is widely recognised. An international Delphi study identified 11 major referral criteria for out-patient SPC referral [30] although these require further validation and customisation. The use of cancer-specific 'transition points' has been proposed to help integrate palliative care more into routine oncological care [31]. The transition points relate to factors associated with poor prognosis (e.g. first hospital admission with metastatic disease). Palliative care referral, however, still tended to be late in the course of the disease (often less than 30 days prior to death) [31]. The importance of understanding the needs of particular cancer sub-populations is recognised to help determine the most appropriate care model [32].

Hence, this study's finding of 'critical moments' in the disease trajectory is very pertinent. In particular, defining the role of SPC within the initial decisionmaking period would be beneficial, especially for those recognised to be at 'high risk' of developing recurrent disease. A partnership model of care was emphasised as integral by healthcare professionals, representing a more gradual transition from one team to another. This is noteworthy as service issues can arise when integration between teams is lacking for those caring for HNC patients [33]. A broad MDT approach is needed to best optimise care, in particular focusing on information and psycho-social needs.

The fact there were misconceptions about hospices and palliative care is not a new issue but was recognised within this study as a fundamental barrier for effective communication. It is acknowledged there are strongly held public attitudes about death and dying [34]. Additionally, barriers perceived by healthcare professionals about discussing palliative care include the risk of causing upset or removing hope [35]. The importance of healthcare professionals having 'upstream' conversations about palliative care (i.e. earlier in the disease trajectory, before individuals actually need the service) has been emphasised [36]. This facilitates an opportunity to understand what the patient and/or family already knows about palliative care, enables time for addressing any misconceptions and allows discussions to be staged overtime [36].

Strengths and limitations

This study has several strengths. Firstly, data from patients, family carers, and healthcare professionals enabled multiple perspectives to be obtained and brought breadth and depth to the study [37]. Additionally, we had a diverse group of patients in terms of the primary site of disease; the age range; and their interaction with different components of SPC clinical services.

There were limitations to the study. Firstly, although we obtained a wide range of views, the study is relatively small-scale and only four family carers were recruited. Secondly, we did not recruit any patients who had been treated with curative intent, were recognised to be 'high risk' for recurrence, but had not yet received SPC input. Their viewpoint could have helped provide further information about palliative care perceptions, ideas about the optimal way to address

Page $5 / 9$ 
any misconceptions and suggest solutions to help enhance integration. The reasoning for this may be related to a number of factors. The definition of 'advanced cancer' may have been too ambiguous compared with using a specific Tumour Node Metastasis (TNM) classification. Alternatively, there may have been a degree of healthcare professional 'gatekeeping'. Potentially, there was a reluctance to consider those who weren't already linked into SPC services in case participation caused distress. Additionally, there was a reliance on the clinical team to establish initial contact with the potential participant. Time and work pressures may have impacted on the ability for clinicians to undertake these actions.

Finally, due to real difficulties with verbal communication, one of the patient interviews was very short. This patient, along with one other, used written communication to support their interview. Despite this limitation, this reflects the 'real world' or practical issues for those affected by advanced HNC. Limiting the study to only those who were verbally articulate did not seem ethically appropriate and instead an inclusive approach to engagement was adopted. These challenges reflect the pragmatic constraints of qualitative research in 'hard to reach' groups.

Implications and unanswered questions

Longitudinal serial interviews provide additional insights into both the patient and family journey [38, 39]. Conducting such a study for those identified as having a 'high risk' of developing recurrent HNC would be beneficial to further understand and learn from these experiences, especially as decision-making is recognised to be so complex. Defining, developing and then targeting interventions at the identified 'critical moments' seems pertinent to help further improve care for HNC patients and their families. Clarifying the balance between how much palliative care should be provided by the wider HNC MDT and how much by SPC services is important to ensure equity and sustainability [40]. The use of decision-support tools and an enhanced patient-centred approach may be one method to help integration [34]. Additionally, the pivotal role of the Clinical Nurse Specialist requires further definition. This may contribute to research exploring the role of oncology nurses to help address symptom needs, coordinate care and offer advance care planning [41].

\section{Conclusion}

Conceptually, the periods highlighted as 'critical moments' in the patients' disease trajectory should be a focus for future interventions to help improve and support care. In particular, these interventions should direct improvements in the provision of information to help inform decision-making and psychological support. Models of palliative care for this population need to be developed in partnership with existing services to ensure care is delivered in a coordinated and timely manner.

\section{Abbreviations}

HNC - head and neck cancer

MDT - Multi-disciplinary Team

SPC - Specialist Palliative Care

UK - United Kingdom

\section{Declarations}

Ethics approval and consent to participate

Ethical approval was obtained from the Health Research Authority and the North West -Greater Manchester West Research Ethics Committee (REC 17/NW/0083; IRAS project ID 221772). All procedures performed in studies involving human participants were in accordance with the ethical standards of the institution and/or national research committee and with the 1964 Helsinki declaration and its later amendments or comparable ethical standards. All data were stored in accordance with the National Health Service (NHS) and University data management and storage policies. Informed consent was obtained from all individual participants included in the study.

Consent for publication

Informed consent was obtained from all individual participants included in the study and specifically included consent to use anonymised quotations in publications.

Availability of data and material

The datasets generated and analysed during the current study are predominately qualitative in nature and are available from the corresponding author on reasonable request.

Competing interests

The authors declare that they have no completing interests.

Funding

Funding for this study was provided by Liverpool Clinical Commissioning Group 'Research Capability Funding'. This funder did not have a role in the study design, collection, analysis or interpretation of the data nor in the writing of the manuscript. Charitable funding was also provided by the Aintree Head and 
Neck Patient and Carer Research Forum. Mr Dominic Macreavy, a co-author for this manuscript, is the Chair of this Forum.

Authors contributions

CRM, SNR, AG, and BJ conceived and designed the study with specific contributions from SM, $\mathrm{CH}$ and DM. HCD conducted the qualitative interviews. CRM, HD and $B J$ analysed the data. CRM, HCD, SNR, AG, SM, CH, DM, BAJ interpreted the data. CM drafted the manuscript and all authors have approved the submitted version.

Acknowledgements

We wish to thank all the participants for their time and contribution to the interviews, and to Helen Rowe for her support with helping transcribe the interviews. Additionally, we would like to thank the support provided from the research sites and participant identification centres.

\section{References}

1. Jemal A, Bray F, Center MM, et al. Global cancer statistics. CA Cancer J Clin 2011; 61:69.

2. So WK, Choi KC, Chen JMT, Chair SY and Fung OWM. Quality of life in head and neck survivors at 1 year after treatment: the mediating role of unmet supportive care needs. Support Care Cancer 2014; 22: 2917-2926.

3. Callahan, C. Facial disfigurement and sense of self in head and neck cancer. Social Work in Health Care 2004; 40 (2): 73-87.

4. Ferlito A, Rogers SN, Shaha AR, Bradley PJ and Rinando A. Quality of Life in Head and Neck Cancer. Acta Otolaryngol 2003; $123:$ 5-7.

5. Lang H, France E, Williams $\mathrm{G}$ et al. The psychological experience of living with head and neck cancer: a systematic review and meta-synthesis. Psychooncology 2013; 22: 2648-2663.

6. Kam D, Salib A, Gorgy G, Patel TD, Carniol ET, Eloy JA, Baredes S, Park RC. Incidence of suicide in patients with head and neck cancer. JAMA Otolaryngol Head Neck Surg 2015; 141 (12): 1075-81.

7. Badr H, Herbert K, Reckson B et al. Unmet needs and relationship challenges of head and neck cancer patients and their spouses. J Psychosoc Oncol. 2016 Jul-Aug; 34(4): 336-346.

8. Chandylen L, Lagorio L, Carnaby G. A Prospective Pilot Study of Psychosocial Functioning in Head and Neck Cancer Patient-Caregiver Dyads. J Psychosoc Oncol. 2014;32(5):477-92. doi: 10.1080/07347332.2014.936649.

9. Balfe M, Keohane K, O’Brien K et al. Social networks, social support and social negativity: A qualitative study of head and neck cancer caregivers' experiences. Eur J Cancer Care 2017; 26 (6) doi: 10.1111/ecc.12619. Epub 2016 Dec 22

10. Hui D, Hannon BL, Zimmerman C et al. Improving patient and caregiver outcomes in oncology: team-based, timely and targeted palliative care. Ca Cancer J Clinc 2018; 68: 356-376.

11. Ferrell B, Sun V, Hurria A et al. Interdisciplinary Palliative Care for Patients with Lung Cancer. J Pain Symptom Manage 2015; 50 (6): $758-67$.

12. Temel J, Greer J, Muzikansky A et al. Early palliative care for patients with metastatic non-small cell lung cancer. N Eng J Med 2010; 363 (8): $733-42$.

13. Bakitas MA, Tosteson TD, Li Z et al. Early versus delayed initaiton of concurrent palliative oncology care: Patient outcomes in the ENABLE III randomised controlled trial. J Clin Oncol 2015; 33 (13): 1438-45.

14. Zimmerman C, Swami N, Krzyzanowska M et al. Early palliative care for patients with advanced cancer: a cluster-randomised controlled trial. Lancet 2014; 383 (9930): 1721-30.

15. Haun MW, Estel S, Rucker G et al. Early palliative care for adults with advanced cancer. Cochrane systematic review (published 12 June 2017 ). Cochrane library. https://www.cochrane.org/CD011129/SYMPT_early-palliative-care-adults-advanced-cancer (last accessed 12th June 2019)

16. Ferrell BR, Temel TS, Temin S et al. Integration of palliative care into standard oncology care: American Society of Clinical Oncology clinical practice guideline update. J Clin Oncol 2017: 35 (1): 96-112.

17. Hui D, Cherny N, Latino F et al. Characteristics and level of integration of ESMO Designated Centres of integrated oncology and palliative care. Annals of Oncology 2016; 27 (6): 1301PD

18. Schenker Y, Robert MA, Bauman JE et al. An enhanced role for palliative care in multidisciplinary approach for high risk head and neck cancer. Cancer 2016; 122 (3): 340-343.

19. Hughes C, Homer J, Bradley P et al. An evaluation of current services available for people diagnosed with head and neck cancer in the UK (2009 - 2010). Clin Oncol 2012; 14: e187-e192.

20. Palliative and supportive care in head and neck cancer: United Kingdom National Multidisciplinary Guidelines. H Cocks, K Ah-See, M Capel, and P Taylor. J Laryngol Otol. 2016 May; 130(Suppl 2): S198-S207. doi: 10.1017/S0022215116000633 (https://www.cambridge.org/core/services/aop-cambridgecore/content/view/543AF37F96D5A88E8123EA86320F792F/S0022215116000633a.pdf/palliative_and_supportive_care_in_head_and_neck_cancer_unite (last accessed 12th June 2019)

21. Ritchie, J. and Lewis. J. (eds.) (2003) Qualitative Research Practice: A Guide for Social Science Students and Researchers. Sage Publications, London.

22. Polit DF and Beck C (2017) Essentials of nursing research. Appraising evidence for nursing practice (9 $9^{\text {th }}$ edition) Wolters Kluwer Health, Philadelphia.

23. NHS England. NHS England Specialist Level Palliative Care: Information for commissioners April 2016. https://www.england.nhs.uk/wpcontent/uploads/2016/04/speclst-palliatv-care-comms-guid.pdf (last accessed 12th June 2019)

24. Miles M \& Huberman A (1994) Qualitative Data Analysis: An expanded sourcebook, Sage London pp 27-34 ('Sampling: Bounding the collection of data'). 
25. Malterud K, Siersma, Guassora AD. Sample size in qualitative interview studies: guided by information power. Qual Health research 2016; 26 (13): 17531760.

26. Oken MM, Creech RH, Tormey DC et al. Toxicity and response criteria of the Eastern Cooperative Oncology Group. Am J Clin Oncol 1982; 5: 649-55.

27. Musselwhite K, Cuff L, McGregor L and King K (2007). The telephone interview is an effective way of data collection in clinical nursing research: a discussion paper. International Journal of Nursing Studies; 44 (6): 1064-1070.

28. Irvine A (2010). Realities toolkit 14. Using phone interviews. Realities part of the ESRC National Centre for Research Methods http://eprints.ncrm.ac.uk/1576/1/14-toolkit-phone-interviews.pdf

29. Colazzi P. Psychological research as the phenomenologist views it. In: R Vale and M Kings (eds): Existential Phenomenological Alternatives for Psychology Oxford University Press; 1978 New York USA pages 48-71.

30. Hui D, Mori M, Watanabe SM et al. Referral criteria for outpatient specialist palliative care: an international consensus. Lancet Oncol 2016; 17 (12): e5529.

31. Collins A, Sundarararajan V, Burchell $\mathrm{J}$ et al. Transition points for the routine integration of palliative care in patients with advanced cancer. $\mathrm{J}$ Pain Symptom Manage 2018; 56 (2): 185 - 194.

32. Temel JS, Greer JA, El-Jawahri A et al. Effects of early integrated palliative care in patients with lung and GI cancer: a randomised clinical trial. J Clin Oncol. 2017 Mar 10;35(8):834-841. doi: 10.1200/JC0.2016.70.5046. Epub 2016 Dec 28.

33. Ullgren $\mathrm{H}$, Kirkpatrick L, Kilpelainen S, Sharp L. Working in silos? Head and neck cancer patients during and after treatment with or without early palliative care referral. Eur J Oncol Nurs 2017; 26: 56-62

34. Cox K, Bird L, Arthur A et al. Public attitudes to death and dying in the UK: a review of published literature. BMJ Support Palliat Care 2013 ; 3 (1): $37-45$.

35. Le BH, Mileshkin L, Doan K et al. Acceptability of early integration of palliative care in patients with incurable lung cancer. J Palliat Med 2014; 17 (5): $553-$ 558.

36. Collins A, McLachlan S-A and Philip J. How should we talk about palliative care, death and dying? A qualitative study exploring perspectives from caregivers of people with advanced cancer. Palliat Med 2018; 32 (4): 861-869.

37. Denzin NK \& Lincoln YS (Eds) (2000). Handbook of qualitative research ( $2^{\text {nd }}$ edition). Sage Publications London.

38. Molassiotis A and Rogers M. Symptom experience and regaining normality in the first year following diagnosis of head and neck cancer: a qualitative longitudinal study. Pall Support Care 2012; 10: 197-204.

39. Isaksson J, Salander P, Lilliehorn S et al. Living an everyday life with head and neck cancer 2-2.5 years after diagnosis - a qualitative prospective study of 56 patients. Social Science and Medicine 2016; 154: 54-61.

40. Quill TE and Abernethy AP. Generalist plus specialist palliative care - creating a more sustainable model. N Eng J Med 2013 ; 368 (13): $1173-5$

41. Robbins-Welty GA, Mueser L, Mitchell C et al. Interventionalist training and intervention fidelity monitoring and maintenance for CONNECT, a nurse-led primary palliative care in oncology trial. Contem Clin Trials Commun 2018; 10:57-61.

\section{Figures}


Figure 1: Flow diagram illustrating patient recruitment

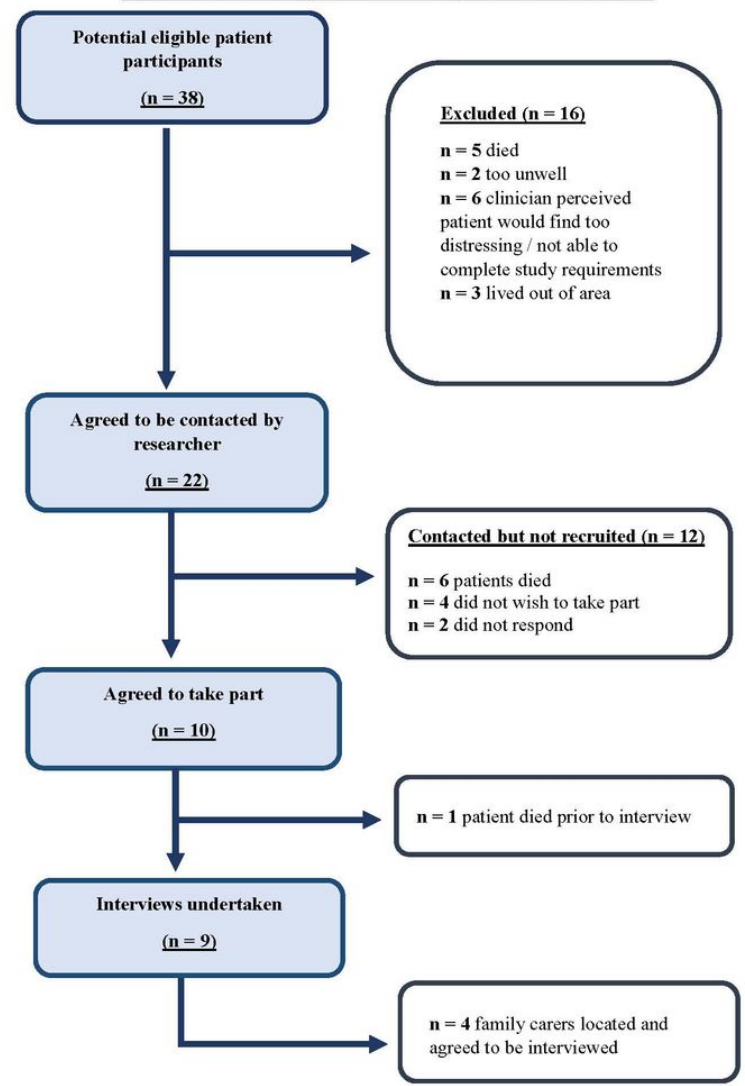

\section{Figure 1}

Flow diagram illustrating patient recruitment

\section{Supplementary Files}

This is a list of supplementary files associated with this preprint. Click to download.

- supplement1.docx 